\title{
Simulating the dynamical quantum Hall effect with superconducting qubits
}

\author{
Xu-Chen Yang, ${ }^{1,2}$ Dan-Wei Zhang, ${ }^{1,3,{ }^{*}}$ Peng Xu, ${ }^{2}$ Yang Yu, ${ }^{2,4}$ and Shi-Liang Zhu ${ }^{2,4, \dagger}$ \\ ${ }^{1}$ Laboratory of Quantum Engineering and Quantum Materials, SPTE, South China Normal University, Guangzhou 510006, China \\ ${ }^{2}$ National Laboratory of Solid State Microstructures and School of Physics, Nanjing University, Nanjing 210093, China \\ ${ }^{3}$ Department of Physics and Center of Theoretical and Computational Physics, The University of Hong Kong, \\ Pokfulam Road, Hong Kong, China \\ ${ }^{4}$ Synergetic Innovation Center of Quantum Information and Quantum Physics, \\ University of Science and Technology of China, Hefei 230026, China
}

(Received 6 October 2014; revised manuscript received 28 October 2014; published 5 February 2015)

\begin{abstract}
We propose an experimental scheme to simulate the dynamical quantum Hall effect and the related interactioninduced topological transition with a superconducting-qubit array. We show that a one-dimensional Heisenberg model with tunable parameters can be realized in an array of superconducting qubits. The quantized plateau, which is a feature of the dynamical quantum Hall effect, will emerge in the Berry curvature of the superconducting qubits as a function of the coupling strength between nearest-neighbor qubits. We numerically calculate the Berry curvatures of two-, four-, and six-qubit arrays and find that the interaction-induced topological transition can be easily observed with the simplest two-qubit array. Furthermore, we analyze some practical conditions in typical experiments for observing this dynamical quantum Hall effect.
\end{abstract}

DOI: 10.1103/PhysRevA.91.022303

\section{INTRODUCTION}

The quantum Hall effect (QHE) is one of the most remarkable phenomena in condensed matter physics [1,2]. The basic experimental fact characterizing QHE is that the nondiagonal conductivity is quantized in the form of $n e^{2} / h$, with $n$ being an integer (the integer QHE) or a fractional number (the fractional QHE). The integer $n$ is a topological invariant which can be expressed as the integral of the Berry curvature [3] over the momentum space $[4,5]$. The Berry curvature and its associated Berry phase have many additional applications in condensed matter physics [6,7] and quantum computation [8-10]. Usually the Berry phase is measured with the interference experiments. Recently, it was proposed that the Berry curvature and hence the Berry phase in generic systems can be detected as a nonadiabatic response on physical observables to the rate of change of an external parameter $[11,12]$. This phenomenon can be interpreted as a dynamical QHE in a parameter space, while the conventional QHE is a particular example of the general relation if one views the electric field as a rate of change of the vector potential [11]. This work opens up the possibility of studying the QHE in parameter space and measuring the Berry phase in many-body systems.

On the other hand, superconducting qubits have become one of the leading systems for study of the Berry phase and simulation of some interesting phenomena that have emerged in condensed matter physics [13]. The Berry phase [14], non-Abelian nonadiabatic geometric gates [15], and geometric Landau-Zener interference [16] were experimentally demonstrated with superconducting qubits. Furthermore, a topological transition characterized by a change in the Chern number was also experimentally observed $[17,18]$. These studies suggest that the superconducting-qubit system is a promising one for further exploration of the rich topological features of single-particle and many-body physics.

\footnotetext{
*zdanwei@126.com

†slzhu@nju.edu.cn
}

PACS number(s): 03.67.Ac, 03.65.Vf, 73.43.-f, 74.81.Fa
In this paper, we propose an experimental scheme to simulate the dynamical QHE and the related interactioninduced topological transition with a superconducting-qubit array. The one-dimensional (1D) Heisenberg spin chain was proposed for realization with superconducting qubits $[19,20]$. We first extend this approach to show that an almost-isotropic interaction [i.e., $J_{j}^{x}=J_{j}^{y} \approx J_{j}^{z}$ in Eq. (4)] between nearestneighbor superconducting qubits can be achieved by coupling phase qubits with the Josephson junctions controlled with the bias current. One of the advantages of the system is that all parameters in this 1D Heisenberg model are controllable and tunable in experiments. We then show that the dynamical QHE and the related interaction-induced topological transition can be observed in the system. We numerically calculate the Berry curvatures of two-, four-, and six-qubit arrays and find that the interaction-induced topological transition can be easily observed with the simplest two-qubit array. We also discuss some practical conditions for observing the dynamical QHE in this system, such as the limit of the ramp velocity, the control errors in experiments, and the decoherence effects for realistic open-system conditions.

The rest of this paper is organized as follows: Section II introduces our proposed superconducting phase qubit array and the realization of the required spin Hamiltonian. Section III presents our results for observing the dynamical QHE and the related interaction-induced topological transition in the proposed system. In Sec. IV, we analyze the ramp velocity limit, the robustness of our scheme against the control errors, and the decoherence effects for realistic conditions and, finally, present our conclusions.

\section{SYSTEM AND HAMILTONIAN}

It was demonstrated that the dynamical QHE can emerge in a 1D Heisenberg spin-chain model with tunable parameters [11]. We consider the 1D Heisenberg spin-chain model with an external magnetic field,

$$
H=-\sum_{j=1}^{N} \vec{h} \cdot \vec{\sigma}_{j}+J \sum_{j=1}^{N-1} \vec{\sigma}_{j} \cdot \vec{\sigma}_{j+1},
$$




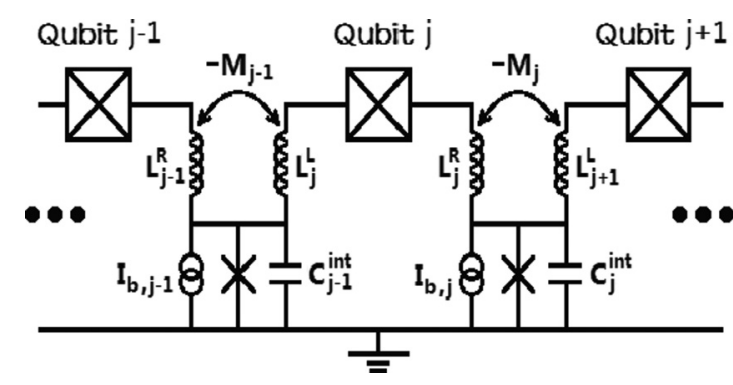

FIG. 1. Schematic of the superconducting-qubit array to simulate the dynamical QHE. The nearest-neighbor phase qubits, such as qubit $j$ and qubit $j+1$, are coupled through a Josephson junction (denoted $\mathrm{X}$ ) with capacitance $C_{j}^{\text {int }}$. The two qubits also contain the capacitances $C_{j}$ and $C_{j+1}$, while the circuit has a negative mutual inductance, $-M_{j}$, for the inductors $L_{j}^{R}$ and $L_{j+1}^{L}$ and a tunable bias current, $I_{b, j}$.

where $\vec{\sigma} \equiv\left(\sigma_{x}, \sigma_{y}, \sigma_{z}\right)$ stands for Pauli matrices, $J$ is the isotropic coupling constant between the nearest-neighbor spins, $\vec{h} \equiv\left(h^{x}, h^{y}, h^{z}\right)$ is the external magnetic field, and $N$ is the size of the spin chain. In the following, we show that this Hamiltonian with tunable coupling constants can be realized in an array of superconducting phase qubits and the related dynamical QHE can be observed in this system.

A schematic of the whole system we consider is shown in Fig. 1. It is an array of $N$ superconducting phase qubits coupled with Josephson junctions. Phase qubit $j$ constitutes an "atom-like" two-level system. The truncated Hamiltonian of the lowest two levels $(|0\rangle,|1\rangle)$ in the energy bases is $H_{q}=$ $\frac{1}{2} \hbar \omega_{q} \sigma_{z}$, where $\hbar \omega_{q}$ represents the energy difference between $|0\rangle$ and $|1\rangle$ and $\sigma_{z}$ is the Pauli operator in the $z$ direction [16,21]. For simplicity, we assume the same parameters for all phase qubits (i.e., $\omega_{q, j}=\omega_{q}$ ). Moreover, the state of each qubit can be controlled by microwaves. In the rotating frame of an applied microwave with frequency $\omega_{d}$, the Hamiltonian for the qubits can be written as $[13,20]$

$$
H=-\sum_{j=1}^{N} \vec{h} \cdot \vec{\sigma}_{j}+H_{\mathrm{int}} .
$$

Here the interacting part of the Hamiltonian $H_{\text {int }}$ is addressed later, and $\vec{h}$ is an effective magnetic field induced by the microwave and can be parameterized as [16-18]

$$
\begin{aligned}
& h^{x}(t)=h \sin \theta \cos \phi, \\
& h^{y}(t)=h \sin \theta \sin \phi, \\
& h^{z}(t)=h \cos \theta .
\end{aligned}
$$

Here the parameter $\phi$ represents the phase of the applied microwave, $h \sin \theta$ is the Rabi oscillation frequency proportional to the amplitude of the microwave, and $h \cos \theta=\omega_{d}-\omega_{q}$ is the detuning, with $\omega_{d}$ being the frequency of the microwave and $\theta$ being the mixing angle. The mixing angle is used as the ramping parameter for observing the dynamical QHE in the following.

As shown in Fig. 1, the interaction between nearestneighbor qubits $j$ and $j+1$ is realized by the inductances $L_{j}^{R}$ and $L_{j+1}^{L}$ and the Josephson junction characterized by capacitance $C_{j}^{\text {int }}$. The two qubits also contain the capacitances $C_{j}$ and $C_{j+1}$, while the circuit has a negative mutual inductance, $-M_{j}$, and a tunable bias current, $I_{b, j}$. Thus in this system, the coupling strengths can be tuned via the bias current of the coupled Josephson junctions $I_{b, j}$, and the Hamiltonian of the interacting part $H_{\text {int }}$ can be written as $[19,20]$

$$
H_{\mathrm{int}}=\sum_{j=1}^{N-1}\left(J_{j}^{x} \sigma_{j}^{x} \sigma_{j+1}^{x}+J_{j}^{y} \sigma_{j}^{y} \sigma_{j+1}^{y}+J_{j}^{z} \sigma_{j}^{z} \sigma_{j+1}^{z}\right),
$$

where the coupling strengths along the three spin directions are, respectively, given by [19]

$$
\begin{aligned}
& J_{j}^{x}=J_{j}^{y}=\frac{\tilde{M}_{j}-\tilde{L}_{j}^{\mathrm{int}} /\left[1-\left(\omega_{q} \omega_{j}^{\mathrm{int}}\right)^{2}\right]}{\tilde{L}_{j}^{R} \tilde{L}_{j+1}^{L} \omega_{q} \sqrt{C_{j} C_{j+1}}}, \\
& J_{j}^{z}=\frac{1}{6 \sqrt{N_{1, j} N_{2, j}}} \frac{\tilde{M}_{j}-\tilde{L}_{j}^{\mathrm{int}}}{\tilde{L}_{j}^{R} \tilde{L}_{j+1}^{L} \omega_{q} \sqrt{C_{j} C_{j+1}}} .
\end{aligned}
$$

Here $\tilde{L}_{j}^{\text {int }}=L_{j}^{\text {int }}\left(1+\frac{M_{j}}{L_{j}^{R}}\right)\left(1+\frac{M_{j}}{L_{j+1}^{L}}\right)$ and $\omega_{j}^{\text {int }}=1 / \sqrt{L_{j}^{\text {int }} C_{j}^{\text {int }}}$, with $L_{j}^{\text {int }}=1 / \sqrt{I_{j, \mathrm{cr}}^{2}-I_{b, j}^{2}}$ and $I_{j, \mathrm{cr}}$ being the critical current of the inter-Josephson junction, the renormalization parameters (mutual and coupling inductances) are $\frac{\tilde{M}_{j}}{M_{j}}=\frac{\tilde{L}_{j}^{R}}{L_{j}^{R}}=\frac{\tilde{L}_{j+1}^{L}}{L_{j+1}^{L}}=$ $1-\frac{M_{j}^{2}}{L_{j}^{R} L_{j+1}^{L}}$, and $N_{1, j}\left(N_{2, j}\right)$ is crudely the number of energy levels in the energy potential well for qubit $j(j+1)$, which is about 5 in typical experiments with phase qubits.

From Eqs. (5) and (6), we calculate the coupling coefficients $J_{j}^{x}$ and $J_{j}^{z}$ for the typical homogeneous parameters $\omega_{q}=$ $4.77 \mathrm{GHz}, C_{j}=1.0 \mathrm{pF}, L_{j}=0.7 \mathrm{nH}, L_{j}^{L}=L_{j}^{R}=3.0 \mathrm{nH}$, $M_{j}=0.41 \mathrm{nH}, N_{1, j}=N_{2, j}=5$, and $I_{j, \mathrm{cr}}=3.0 \mu \mathrm{A}$ [19]. In this case, we get homogeneous (qubit-independent) coupling strengths and thus the qubit label $j$ in the coupling strengths $J_{j}^{r}(r=x, y, z)$ is omitted hereafter, for simplicity. The results are plotted in Fig. 2, showing that if we adjust the bias current from 0 to $0.93 I_{j, \mathrm{cr}}$, the coupling strength $J^{x}$ will continuously and monotonically decrease from about $40 \mathrm{MHz}$ to 0 . Furthermore, when the bias current is less than the critical

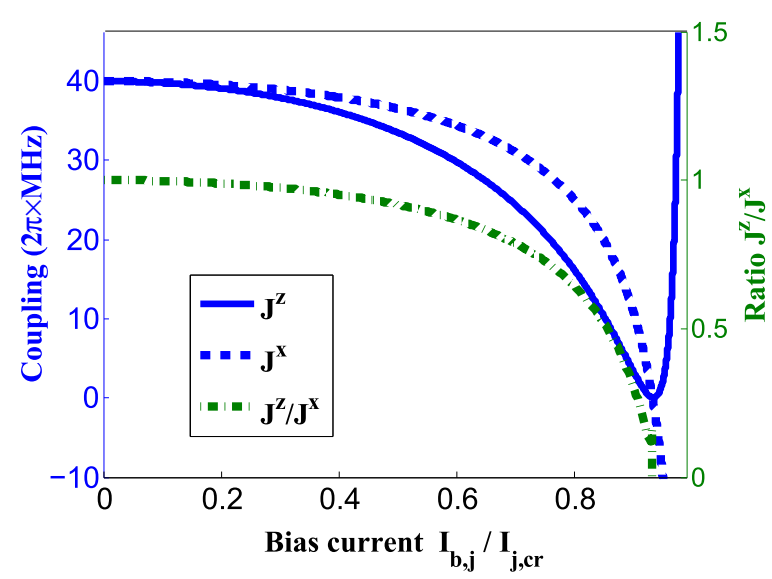

FIG. 2. (Color online) The strength of coupling $J^{x}$ [dashed (blue) line] and $J^{z}$ [solid (blue) line] and the ratio $J^{z} / J^{x}$ [dashed-dotted (green) line] as functions of the bias current $I_{b, j} / I_{j, \mathrm{cr}}$. The typical parameters are $\omega_{q}=4.77 \mathrm{GHz}, C_{j}=1.0 \mathrm{pF}, L_{j}=0.7 \mathrm{nH}, L_{j}^{L}=$ $L_{j}^{R}=3.0 \mathrm{nH}, M_{j}=0.41 \mathrm{nH}, N_{1, j}=N_{2, j}=5$, and $I_{j, \mathrm{cr}}=3.0 \mu \mathrm{A}$. 
current $0.54 I_{j, \text { cr }}$ the ratio $J^{z} / J^{x}$ remains in the region about $[0.9,1]$. As we show in the following, this parameter region already allows observation of the dynamical QHE.

\section{SIMULATING DYNAMICAL QUANTUM HALL EFFECT}

The topological features of the superconducting-qubit system can be probed by measuring the Berry curvature, while Fig. 3 depicts a typical sequence used to measure the Berry curvature. To demonstrate the dynamical QHE in this system, we follow the proposal in Ref. [11] to analyze the quantized response of the system to a rotating magnetic field. We consider all the superconducting qubits initially in the ground state with $\theta(t=0)=0$ and then ramp the system with fixed $\phi(t)=0$ to undergo a quasiadiabatic evolution by varying the mixing angle $\theta(t)=v^{2} t^{2} / 2 \pi$ for a ramp time $t_{\text {ramp }}=\pi / v$, where $v$ denotes the ramp velocity. At the end of such a ramp, the velocity of the $\theta$ component of the magnetic field $v_{\theta}(t)$ is exactly $v$, and we can measure the Berry curvature of the system. We note that this choice of ramping field guarantees that the angular velocity is turned on smoothly and the system is not excited at the beginning of the evolution [11].

The three components of the effective magnetic field $h^{x, y, z}$ during the ramping process are depicted in Fig. 3(a). The generalized force for the full Hamiltonian $H$, which is measured at $t=t_{\text {ramp }}$, is along the latitude direction (at the point of measurement it is along the $y$ axis) and given by

$$
M_{\theta}=-\left.\left\langle\partial_{\phi} H\right\rangle\right|_{\phi=0, t=\pi / v}=h \sum_{j=1}^{N}\left\langle\sigma_{j}^{y}\right\rangle,
$$

while the quench velocity is along the longitude direction. Then we can obtain the Berry curvature $F_{\theta \phi}$ within the linear response approximation $[11,12]$,

$$
F_{\theta \phi}=\frac{M_{\theta}}{v_{\theta}}=\frac{h}{v} \sum_{j=1}^{N}\left\langle\sigma_{j}^{y}\right\rangle .
$$

In experiments, one can measure the $\left\langle\sigma_{j}^{y}\right\rangle$ of each superconducting qubit, and then the Berry curvature can be derived by substituting the results into Eq. (8). In other words, the qubit system in the described evolution progress is initially prepared

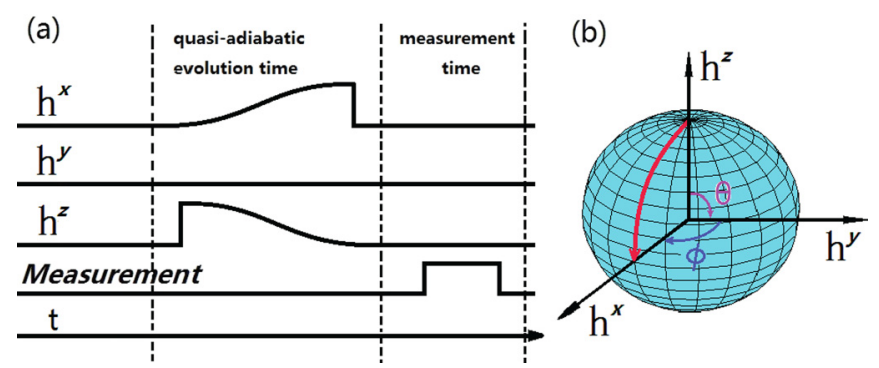

FIG. 3. (Color online) (a) Schematic sequence of the parameter evolution. Superconducting qubits undergo a nonadiabatic evolution, with the amplitude of the effective magnetic field strength followed by Eq. (3). Then the $\left\langle\sigma_{j}^{y}\right\rangle$ of the final state is measured. (b) Schematic of the effective magnetic-field strength. The solid (red) curve represents the evolution path. in the ground state and then slowly and smoothly driven along the $\theta$ direction, as shown in Fig. 3(b). The generalized force $M_{\theta}$ along the orthogonal direction is measured as a linear response to the ramping magnetic field. We see the quantization of this response in the following, and in this sense it is called the dynamical QHE [11].

The simplest system for observing the dynamical QHE and its related interaction-induced topological transition should be a two-qubit system. Therefore we first address whether one can observe this phenomenon in an array with two superconducting qubits. To this end, we numerically calculate the Berry curvature $F_{\theta \phi}$ in a two-qubit array as a function of the bias current $I_{b, j} / I_{j, \text { cr }}$ for the described ramp process by time-dependent exact diagonalization [22]. The results are plotted in Fig. 4(a), where the parameters are the same as those in Fig. 2. The solid (red) line in Fig. 4(a) shows
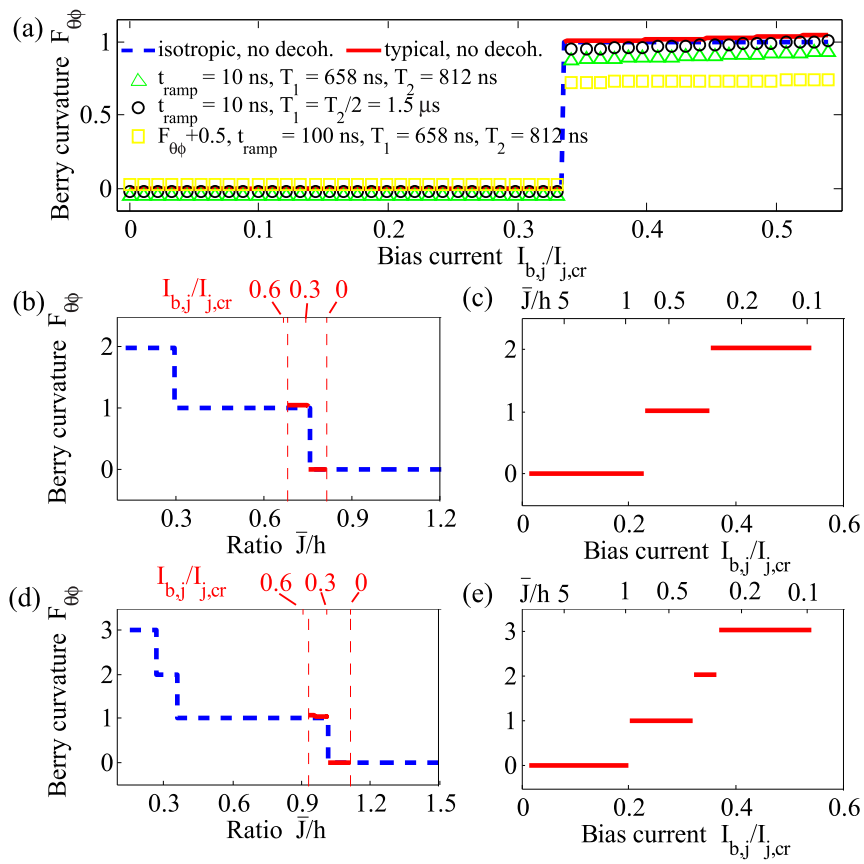

FIG. 4. (Color online) The Berry curvature as a function of the ratio $\bar{J} / h$ (i.e., the ratio of the coupling strength to the amplitude of the effective magnetic field) or the bias current $I_{b, j} / I_{j, \text { cr }}$ in a two-, four-, or six-qubit array. (a) Two-qubit case for $h / 2 \pi=76 \mathrm{MHz}$. The solid (red) line is the Berry curvature as a function of $I_{b, j} / I_{j, \text { cr }}$, and the dashed (blue) line is the result for isotropic coupling strength $\bar{J}=$ $\sqrt{\left(J^{x}\right)^{2}+\left(J^{y}\right)^{2}+\left(J^{z}\right)^{2}} / \sqrt{3}$ (here $J^{x, y, z}$ are determined by $I_{b, j} / I_{j, \text { cr }}$ as shown in Fig. 2). The (green) triangles and (yellow) squares are the corresponding $F_{\theta \phi}\left(I_{b, j} / I_{j, \mathrm{cr}}\right)$ under the open-system conditions for the decoherence times $T_{1}=658 \mathrm{~ns}$ and $T_{2}=812 \mathrm{~ns}$, while the black circles represent the case with $T_{1}=T_{2} / 2=1.5 \mu \mathrm{s}$, with the total measurement time $t_{\text {meas }}=10 \mathrm{~ns}$. (b) Four-qubit case for $h / 2 \pi=$ $49 \mathrm{MHz}$. (c) Four-qubit case for varied $h=-85 \bar{J}+3400 \mathrm{MHz}$. (d) Six-qubit case for $h / 2 \pi=36 \mathrm{MHz}$. (e) Six-qubit case for varied $h=-85 \bar{J}+3400 \mathrm{MHz}$. In (b)-(e), the solid (red) and dashed (blue) lines represent the Berry curvature as a function of $I_{b, j} / I_{j, \text { cr }}$ and $\bar{J} / h$, respectively. The ramp time in (a)-(e) is $t_{\text {ramp }}=100 \mathrm{~ns}$, except that $t_{\text {ramp }}=10 \mathrm{~ns}$ for the (green) triangles and black circles in (a). We note that the results in (a)-(e) for cases without decoherence almost remain for varying $t_{\text {ramp }}$ when $t_{\text {ramp }} \gtrsim 10 \mathrm{~ns}$. 
that although the interaction strengths $J^{x, y, z}$ are not exactly isotropic in our superconducting-qubit system, the plateaus in the Berry curvature are strictly stable at 0 and 1 , and the transition between the two plateaus is very sharp. For comparison, we also calculate the Berry curvature for the isotropic coupling case [dashed (blue) line in Fig. 4(a)], where we choose an isotropic coupling strength $\bar{J}$ determined by $\bar{J}=\sqrt{\left(J^{x}\right)^{2}+\left(J^{y}\right)^{2}+\left(J^{z}\right)^{2}} / \sqrt{3}$ (here $J^{x, y, z}$ depend on the bias current $I_{b, j} / I_{j, \text { cr }}$ as shown in Fig. 2). From Fig. 4(a), it is clear that for the chosen typical parameters the difference in the Berry curvatures between the isotropic and the anisotropic cases can be neglected.

Now we address the dynamical QHE and the interactioninduced topological transition in an $N$-qubit array. For this 1D Heisenberg spin chain, the plateaus in the Berry curvature should appear in an integer $n=0,1,2, \ldots, N / 2$ for an even $N$ [11]. We have numerically confirmed this phenomenon for a four- and a six-qubit array, with $F_{\theta \phi}(\bar{J} / h)$ for typical parameters shown as dashed (blue) lines in Figs. 4(b) and 4(d), respectively. We then further check whether these multiplateaus can be observed in this superconducting-qubit system. For the same corresponding parameters, we calculate the Berry curvature as a function of $I_{b, j} / I_{j, \text { cr }}$ in the region $I_{b, j} / I_{j, \mathrm{cr}} \in$ $[0,0.54]$, which corresponds to the coupling strength in the region $[34,40] \mathrm{MHz}$ as shown in Fig. 2. The results are shown by the solid (red) lines in Figs. 4(b) and 4(d), where we find that only two quantized plateaus with a topological transition appear and other quantized plateaus cannot be observed. One simple approach to solving this problem is to simultaneously change the magnetic field strength $h$ and the bias current $I_{b, j}$. For instance, we can choose $h(\bar{J})=-85 \bar{J}+3400 \mathrm{MHz}$ in simulations, and then the obtained Berry curvatures as a function of $\bar{J} / h$ (and $I_{b, j} / I_{j, \text { cr }}$ ) for four-qubit and six-qubit arrays are shown in Figs. 4(c) and 4(e), respectively. It is clear that all quantized plateaus can be observed in this approach. In the above calculation, the amplitude of $h$ given by the relation equation $h(\bar{J})$ is yet to be optimized and it is about $350 \mathrm{MHz}$ at $\bar{J} / h=0.1$. However, the amplitude of the effective magnetic field required to observe all the quantized plateaus can be much lower after optimization.

\section{DISCUSSION AND CONCLUSIONS}

In the previous calculations, the Berry curvature $F_{\theta \phi}$ is considered to be a linear response to the ramp velocity $v_{\theta}$. In general, the magnetization (the generalized force) is determined by $M_{\theta}=M_{0}+F_{\theta \phi} v_{\theta}+O\left(v_{\theta}^{2}\right)[11,12]$, where the constant term $M_{0}$ gives the value of the magnetization in the adiabatic limit and $M_{0}=0$ in our cases. The linear response theory breaks down when the velocity $v_{\theta}$ is too high to neglect the term related to $v_{\theta}^{2}$. To check the velocity limit in this linear response theory, we numerically calculate the Berry curvature $F_{\theta \phi}$ as a function of the ramp time $t_{\text {ramp }}$ for a two-qubit array, with the results for parameters $\bar{J} / h=0.4$ and $h / 2 \pi=76 \mathrm{MHz}$ plotted in Fig. 5. We can see that the Berry curvature saturates to nearly 1 when $t_{\text {ramp }} \gtrsim 10 \mathrm{~ns}$ and becomes very stable when $t_{\text {ramp }} \gtrsim 60 \mathrm{~ns}$. In addition, the magnetization $M_{\theta}$ is plotted in the inset in Fig. 5 as a function of the finial ramp velocity $v$, which further shows that the linear response approximation works well within $v \lesssim 0.3 \mathrm{rad} / \mu \mathrm{s}$. Therefore,

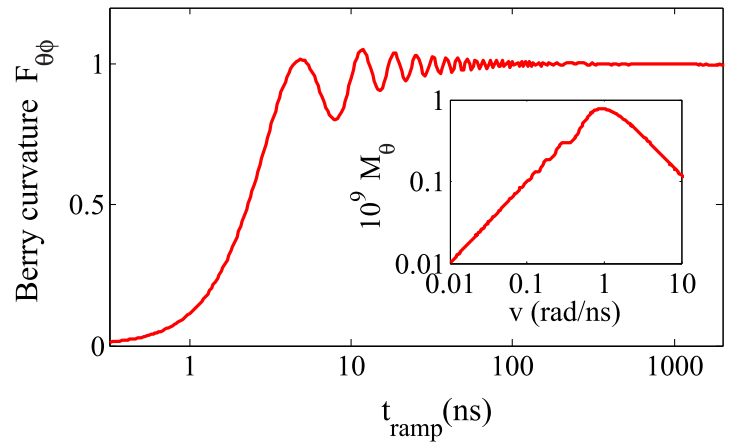

FIG. 5. (Color online) The Berry curvature $F_{\theta \phi}$ as a function of the ramp time for a two-qubit array with isotropic coupling $\bar{J}=0.4 \mathrm{~h}$ and $h / 2 \pi=76 \mathrm{MHz}$. The Berry curvature saturates to nearly 1 when $t_{\text {ramp }} \gtrsim 10$ ns. Inset: Magnetization $M_{\theta}$ as a function of the finial ramp velocity $v$.

to observe the quantized plateaus in Fig. 4(a), the ramp velocity should be slower than $0.3 \mathrm{rad} / \mu \mathrm{s}$, corresponding to a ramp time longer than $10 \mathrm{~ns}$. We also simulate the same procedures for the four-qubit and six-qubit arrays and find that the results are similar to those in Fig. 5. Thus the velocity limit for observation of the quantized plateaus does not change much for arrays with different numbers of qubits.

Then we further study the robustness of the quantized plateaus of the Berry curvature against the control errors which stem from fluctuations of the parameters in Hamiltonian (2). We assume $\tilde{J}^{x, y, x}=\alpha_{1} J^{x, y, z}$ and $\tilde{h}=\alpha_{2} h$, with $\alpha_{1}$ and $\alpha_{2}$ being randomly distributed in the region $[1-\eta, 1+\eta]$ (here $\eta>0$ describes the fluctuation strength). For a single realization with randomly chosen $\alpha_{1}$ and $\alpha_{2}$, we calculate the corresponding $F_{\theta \phi}^{\alpha}$ as that in Fig. 4(a) and then we can obtain the averaged Berry curvature $\bar{F}_{\theta \phi}=1 / N_{\alpha} \sum F_{\theta \phi}^{\alpha}$, where $N_{\alpha}$ denotes the sampling number. The averaged Berry curvature for the two-qubit case as a function of $I_{b, j} / I_{j, \mathrm{cr}}$ is plotted in Fig. 6(a). We find that the plateaus are still stable when the parameter fluctuation strength $\eta$ is less than about $5 \%$, even though their transition is slightly smoothed by the fluctuation. Furthermore, we calculate the corresponding
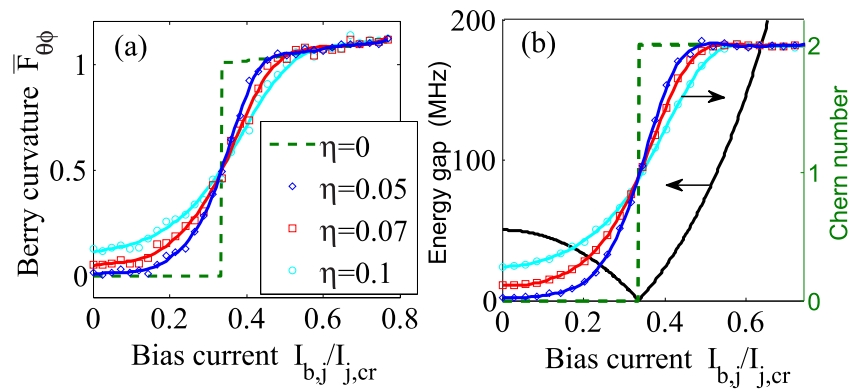

FIG. 6. (Color online) (a) Averaged Berry curvature $\bar{F}_{\theta \phi}$ of two qubits as a function of $I_{b, j} / I_{j, \text { cr }}$ in the presence of fluctuating parameters $\tilde{J}^{x, y, z}$ and $\tilde{h}$. Fluctuation strengths are $\eta=0$ [dashed (green) line], 5\% [(blue) diamonds], 7\% [(red) squares], and 10\% [(cyan) circles]. Other parameters are $h / 2 \pi=76 \mathrm{MHz}$ and $N_{\alpha}=$ 500. The ramp time is $t_{\text {ramp }}=100 \mathrm{~ns}$. (b) The average Chern number $\mathrm{Ch}$ of two qubits with the same parameters as in (a). In addition, the black line denotes the energy gap between the ground state and the first excited state of the system as a function of $I_{b, j} / I_{j, \mathrm{cr}}$. 
Chern number $\mathrm{Ch}=(2 \pi)^{-1} \int_{0}^{\pi} d \theta \int_{0}^{2 \pi} d \phi F_{\theta \phi}=\int_{0}^{\pi} F_{\theta \phi} d \theta$ by integrating the Berry curvature in the $\theta-\phi$ sphere in Fig. 6(b). As we expected, the Chern number is more robust and the quantized plateaus there are more significant due to the averaging over different runs of $\theta$ ramping with parameter fluctuations. In addition, we also plot the energy gap between the ground state and the first excited state of the two-qubit array in Fig. 6(b). We can see that the gap closes at the topological transition point.

We now discuss the decoherence effects in our system for realistic open-system conditions. For simplicity, we assume that each superconducting qubit in the system interacts independently with the environment, which is commonly modeled as a bath of oscillators. The quantum dynamics of the system is thus described by the master equation [23]

$$
\frac{d \rho}{d t}=-\frac{i}{\hbar}[H, \rho]+\sum_{j=1}^{N} \mathcal{L}_{j}[\rho],
$$

where the density matrix $\rho$ is spanned by the $N$-qubit basis, and the Lindblad superoperator $\mathcal{L}_{j}[\rho]$ describes the decoherence due to the independent interaction between each qubit and the bath. We further assume a weak qubit-bath interaction and the Markovian limit and thus the Lindblad superoperator can be written as $\mathcal{L}_{j}[\rho]=\gamma\left(1+n_{0}\right)\left(2 \sigma_{j}^{-} \rho \sigma_{j}^{+}-\left\{\sigma_{j}^{+} \sigma_{j}^{-}, \rho\right\}\right)+$ $\gamma n_{0}\left(2 \sigma_{j}^{+} \rho \sigma_{j}^{-}-\left\{\sigma_{j}^{-} \sigma_{j}^{+}, \rho\right\}\right)+\Gamma\left(2 \sigma_{j}^{z} \rho \sigma_{j}^{z}-\left\{\sigma_{j}^{z} \sigma_{j}^{z}, \rho\right\}\right)$ [23]. Here the first two terms describe the energy relaxation progress with parameter $\gamma$, the third term describes the pure dephasing progress with parameter $\Gamma$, and the effective boson number $n_{0}$ on each qubit depends on the temperature of the bath $T$, with $n_{0}=1 /\left[\exp \left(\hbar \omega_{q} / k_{B} T\right)-1\right]$ (here $k_{B}$ is the Boltzmann constant). In the superconducting-qubit system, we have $n_{0} \approx$ 0 because $\hbar \omega_{q} \gg k_{B} T$ for $T \approx 30 \mathrm{mK}$ and $\omega_{q}$ is of the order of gigahertz in practical experiments [16-18]. Then the usually measured relaxation time $T_{1}$ and dephasing time $T_{2}$ of each qubit are determined by $1 / T_{1}=\gamma$ and $1 / T_{2}=1 / 2 T_{1}+\Gamma$ [23], respectively.

The additional time scale for measurement is another issue one should consider for a finite decoherence time. We assume that each phase qubit in the array can be manipulated and measured independently $[18,24]$. Since the qubits can only be naturally read out in the $\sigma_{z}$ basis (i.e., the $\left\langle\sigma^{z}\right\rangle$ measurement), an additional spin rotation,

$$
\hat{R}=\frac{1}{\sqrt{2}}\left(\begin{array}{cc}
1 & -i \\
-i & 1
\end{array}\right),
$$

for each qubit (effectively an $\hat{X}_{\pi / 2}$ operation in experiments $[17,18])$ has to be inserted in order to measure $\left\langle\sigma_{j}^{y}\right\rangle$ after the ramp. This rotation can be achieved by additional microwave pulses $[16,18]$ of the duration $\tau_{R} \approx \pi / 2 h=3.3 \mathrm{~ns}$ for the cases with $h / 2 \pi=76 \mathrm{MHz}$ in Figs. 4 and 5. Finally, the $\left\langle\sigma_{j}^{z}\right\rangle$ measurement of each qubit requires a duration $\tau_{d}$ which is typically several nanoseconds $[16,24]$. So the total time required for measurement is around $t_{\text {meas }}=\tau_{R}+\tau_{d} \approx 10 \mathrm{~ns}$. Since the measurement fidelity for each phase qubit in the coupled system can be more than 95\% [24], we do not further consider the measurement errors.

To see the decoherence effects in the dynamical QHE in our proposed system, we take the two-qubit array as an example and numerically simulate the whole progression with the ramp and measurement sequences by calculating the master equation (see the Appendix for details). For simplicity in our simulations, we treat the evolution of the qubits over the whole measurement progression with time $t_{\text {meas }}=10 \mathrm{~ns}$ as free evolution under decoherence. In addition, the relaxation time $T_{1}$ and dephasing time $T_{2}$ of single-phase qubits are usually longer than those of multiqubits in the coupled system; this effect is somehow contained in the master equation, which includes the increases in decoherence channels and decoherence rates [see Eq. (A8), Appendix]. We estimate that the effective times $\widetilde{T}_{1}$ and $\widetilde{T}_{2}$ of two qubits are about 5 times shorter than those of a single qubit in the master equation ( $T_{1}$ and $T_{2}$ ), thus we choose typical decoherence times in simulations from single-phase-qubit experiments.

We first take the decoherence times $T_{1} \approx 658 \mathrm{~ns}$ and $T_{2} \approx$ 812 ns of each qubit in experiments on phase qubits [25] as a typical example. From Fig. 5, we know that the linear response condition is satisfied when the ramp time $t_{\text {ramp }} \gtrsim 10 \mathrm{~ns}$. So we numerically calculate the Berry curvature with $t_{\text {ramp }}=10 \mathrm{~ns}$ and the result is plotted as the (green) triangles in Fig. 4(a). In this case, the two plateaus in the Berry curvature $F_{\theta \phi}$ are, respectively, near 0 and 1 (the difference is about 0.96 and the transition point remains), as expected. However, we find that the two plateaus in the Berry curvature are gradually shifted from $F_{\theta \phi}=0$ and 1 when the ramp time becomes longer. For instance, the difference between the two plateaus decreases to about 0.72 for the ramp time $t_{\text {ramp }}=100 \mathrm{~ns}$, which is shown by the (yellow) squares in Fig. 4(a). This is due to the fact that the total evolution time (i.e., $110 \mathrm{~ns}$ ) of the system is now comparable to the effective decoherence times (effective times $\widetilde{T}_{1} \approx 658 / 5$ ns and $\widetilde{T}_{2} \approx 812 / 5 \mathrm{~ns}$ ) and thus the Chern number is no longer a well-defined topological index [26]. Therefore, observation of the dynamical QHE is crucially dependent on the long decoherence time since the Berry curvature (which is associated with the Berry phase factor) has no classical correspondence. To demonstrate the topological features of the dynamical QHE more clearly (or in a longer ramp time), we should improve the coherence time for superconducting qubits in experiments. With the current technology, the relaxation time $T_{1}$ of a phase qubit can be as long as $1.5 \mu$ s [25,27]. One can use dynamical decoupling to increase the dephasing time up to the $T_{2}=2 T_{1}$ limit [28]. Thus, we also numerically calculate the result for $T_{1}=T_{2} / 2=1.5 \mu \mathrm{s}$, and the result is plotted as black circles in Fig. 4(a). It clearly shows that the decoherence effects are almost negligible in this case.

In conclusion, we have proposed an experimental scheme to simulate the dynamical QHE and the related interactioninduced topological transition with a superconducting-qubit array. We find that the typical topological features can even be observed in the simplest two-qubit array under practical experimental conditions.

\section{ACKNOWLEDGMENTS}

We thank Z.-Y. Xue and C.-J. Shan for helpful discussions. This work was supported by the SKPBR of China (Grant No. 2011CB922104), the NSFC (Grants No. 11125417, No. 11474153, and No. 11274156), and the PCSIRT (Grant No. IRT1243). D.W.Z. acknowledges support from an HKU postdoctoral fellowship. 


\section{APPENDIX: THE MASTER EQUATION FOR THE TWO-QUBIT CASE}

In this appendix, we derive the master equation for a twoqubit array with the Hamiltonian

$$
\begin{aligned}
H= & -\sum_{j=1}^{2}\left(h_{j}^{x} \sigma_{x}+h_{j}^{y} \sigma_{y}+h_{j}^{z} \sigma_{z}\right) \\
& +J^{x} \sigma_{1}^{x} \sigma_{2}^{x}+J^{y} \sigma_{1}^{y} \sigma_{2}^{y}+J^{z} \sigma_{1}^{z} \sigma_{2}^{z},
\end{aligned}
$$

where the components of the effective magnetic field $h_{1}^{x, y, z}=$ $h_{2}^{x, y, z}=h^{x, y, z}(t)$ are given by Eq. (3). In the two-qubit basis $\left\{\left|\uparrow_{1} \uparrow_{2}\right\rangle,\left|\downarrow_{1} \uparrow_{2}\right\rangle,\left|\uparrow_{1} \downarrow_{2}\right\rangle,\left|\downarrow_{1} \downarrow_{2}\right\rangle\right\}$, the Hamiltonian matrix can be written as

$$
H=\left(\begin{array}{llll}
H_{11} & H_{12} & H_{13} & H_{14} \\
H_{21} & H_{22} & H_{23} & H_{24} \\
H_{31} & H_{32} & H_{33} & H_{34} \\
H_{41} & H_{42} & H_{43} & H_{44}
\end{array}\right)
$$

where the matrix elements are given by

$$
\begin{aligned}
& H_{11}=-2 h^{z}+J^{z}, \quad H_{22}=H_{33}=-J^{z}, \\
& H_{44}=2 h^{z}+J^{z}, \\
& H_{12}=H_{13}=H_{24}=H_{34}=-h^{x}+i h^{y}, \\
& H_{21}=H_{31}=H_{42}=H_{43}=-h^{x}-i h^{y}, \\
& H_{23}=H_{32}=J^{x}+J^{y}, \quad H_{14}=H_{41}=J^{x}-J^{y} .
\end{aligned}
$$

The quantum dynamics of the system is described by the master equation

$$
\frac{d \rho}{d t}=-i[H, \rho]+\mathcal{L}_{1}[\rho]+\mathcal{L}_{2}[\rho]
$$

where the density matrix $\rho$ is denoted

$$
\rho=\left(\begin{array}{llll}
\rho_{11} & \rho_{12} & \rho_{13} & \rho_{14} \\
\rho_{21} & \rho_{22} & \rho_{23} & \rho_{24} \\
\rho_{31} & \rho_{32} & \rho_{33} & \rho_{34} \\
\rho_{41} & \rho_{42} & \rho_{43} & \rho_{44}
\end{array}\right)
$$

We consider the system in the Markovian and lowtemperature limit, and thus the Lindblad superoperator can be written as

$$
\begin{aligned}
\mathcal{L}_{j}[\rho]= & \gamma\left(2 \sigma_{j}^{-} \rho \sigma_{j}^{+}-\left\{\sigma_{j}^{+} \sigma_{j}^{-}, \rho\right\}\right) \\
& +\Gamma\left(2 \sigma_{j}^{z} \rho \sigma_{j}^{z}-\left\{\sigma_{j}^{z} \sigma_{j}^{z}, \rho\right\}\right)(j=1,2) .
\end{aligned}
$$

Here the relaxation rate $\gamma$ and pure dephsing rate $\Gamma$ are determined by the measured decoherence times: $1 / T_{1}=\gamma$ and $1 / T_{2}=1 / 2 T_{1}+\Gamma$. Using the expansions $\sigma_{1}^{ \pm, z} \rightarrow\left(\sigma^{ \pm, z} \otimes\right.$ $\left.\mathbf{I}_{2 \times 2}\right)$ and $\sigma_{2}^{ \pm, z} \rightarrow\left(\mathbf{I}_{2 \times 2} \otimes \sigma^{ \pm, z}\right)$, one can obtain the Lindblad superoperators:

$$
\mathcal{L}_{1}[\rho]+\mathcal{L}_{2}[\rho]=\left(\begin{array}{cccc}
-4 \gamma \rho_{11} & -(3 \gamma+4 \Gamma) \rho_{12} & -(3 \gamma+4 \Gamma) \rho_{13} & -(2 \gamma+8 \Gamma) \rho_{14} \\
-(3 \gamma+4 \Gamma) \rho_{21} & -2 \gamma\left(\rho_{22}-\rho_{11}\right) & -(2 \gamma+8 \Gamma) \rho_{23} & 2 \gamma \rho_{13}-(\gamma+4 \Gamma) \rho_{24} \\
-(3 \gamma+4 \Gamma) \rho_{31} & -(2 \gamma+8 \Gamma) \rho_{32} & -2 \gamma\left(\rho_{33}-\rho_{11}\right) & 2 \gamma \rho_{12}-(\gamma+4 \Gamma) \rho_{34} \\
-(2 \gamma+8 \Gamma) \rho_{41} & 2 \gamma \rho_{31}-(\gamma+4 \Gamma) \rho_{42} & 2 \gamma \rho_{21}-(\gamma+4 \Gamma) \rho_{43} & 2 \gamma\left(\rho_{22}+\rho_{33}\right)
\end{array}\right)
$$

By substituting Eq. (A7) into Eq. (A4), one can obtain the master equation as

$$
\begin{aligned}
& \dot{\rho}_{11}=-i\left[H_{14}\left(\rho_{41}-\rho_{14}\right)+H_{12} \rho_{21}+H_{13} \rho_{31}-H_{21} \rho_{12}-H_{31} \rho_{13}\right]-4 \gamma \rho_{11}, \\
& \dot{\rho}_{12}=-i\left[\left(H_{11}-H_{22}\right) \rho_{12}+H_{12}\left(\rho_{22}-\rho_{11}\right)+H_{13} \rho_{32}+H_{14} \rho_{42}-H_{32} \rho_{13}-H_{42} \rho_{14}\right]-(3 \gamma+4 \Gamma) \rho_{12} \text {, } \\
& \dot{\rho}_{13}=-i\left[\left(H_{11}-H_{33}\right) \rho_{13}+H_{13}\left(\rho_{33}-\rho_{11}\right)+H_{12} \rho_{23}+H_{14} \rho_{43}-H_{23} \rho_{12}-H_{43} \rho_{14}\right]-(3 \gamma+4 \Gamma) \rho_{13} \text {, } \\
& \dot{\rho}_{14}=-i\left[\left(H_{11}-H_{44}\right) \rho_{14}+H_{14}\left(\rho_{44}-\rho_{11}\right)+H_{12} \rho_{24}+H_{13} \rho_{34}-H_{24} \rho_{12}-H_{34} \rho_{13}\right]-(2 \gamma+8 \Gamma) \rho_{14} \text {, } \\
& \dot{\rho}_{21}=-i\left[\left(H_{22}-H_{11}\right) \rho_{21}+H_{21}\left(\rho_{11}-\rho_{22}\right)+H_{23} \rho_{31}+H_{24} \rho_{41}-H_{31} \rho_{23}-H_{41} \rho_{24}\right]-(3 \gamma+4 \Gamma) \rho_{21} \text {, } \\
& \dot{\rho}_{22}=-i\left[H_{23}\left(\rho_{32}-\rho_{23}\right)+H_{21} \rho_{12}+H_{24} \rho_{42}-H_{12} \rho_{21}-H_{42} \rho_{24}\right]-2 \gamma\left(\rho_{22}-\rho_{11}\right) \text {, } \\
& \dot{\rho}_{23}=-i\left[\left(H_{22}-H_{33}\right) \rho_{23}+H_{23}\left(\rho_{33}-\rho_{22}\right)+H_{21} \rho_{13}+H_{24} \rho_{43}-H_{13} \rho_{21}-H_{43} \rho_{24}\right]-(2 \gamma+8 \Gamma) \rho_{23} \text {, } \\
& \dot{\rho}_{24}=-i\left[\left(H_{22}-H_{44}\right) \rho_{24}+H_{24}\left(\rho_{44}-\rho_{22}\right)+H_{21} \rho_{14}+H_{23} \rho_{34}-H_{14} \rho_{21}-H_{34} \rho_{23}\right]+2 \gamma \rho_{13}-(\gamma+4 \Gamma) \rho_{24} \text {, } \\
& \dot{\rho}_{31}=-i\left[\left(H_{33}-H_{11}\right) \rho_{31}+H_{31}\left(\rho_{11}-\rho_{33}\right)+H_{32} \rho_{21}+H_{34} \rho_{41}-H_{21} \rho_{32}-H_{41} \rho_{34}\right]-(3 \gamma+4 \Gamma) \rho_{31} \text {, } \\
& \dot{\rho}_{32}=-i\left[\left(H_{33}-H_{22}\right) \rho_{32}+H_{32}\left(\rho_{22}-\rho_{33}\right)+H_{31} \rho_{12}+H_{34} \rho_{42}-H_{12} \rho_{31}-H_{42} \rho_{34}\right]-(2 \gamma+8 \Gamma) \rho_{32} \text {, } \\
& \dot{\rho}_{33}=-i\left[H_{23}\left(\rho_{23}-\rho_{32}\right)+H_{31} \rho_{13}+H_{34} \rho_{43}-H_{13} \rho_{31}-H_{43} \rho_{34}\right]-2 \gamma\left(\rho_{33}-\rho_{11}\right) \text {, } \\
& \dot{\rho}_{34}=-i\left[\left(H_{33}-H_{44}\right) \rho_{34}+H_{34}\left(\rho_{44}-\rho_{33}\right)+H_{31} \rho_{14}+H_{32} \rho_{24}-H_{14} \rho_{31}-H_{24} \rho_{32}\right]+2 \gamma \rho_{12}-(\gamma+4 \Gamma) \rho_{34} \text {, } \\
& \dot{\rho}_{41}=-i\left[\left(H_{44}-H_{11}\right) \rho_{41}+H_{41}\left(\rho_{11}-\rho_{44}\right)+H_{42} \rho_{21}+H_{43} \rho_{31}-H_{21} \rho_{42}-H_{31} \rho_{43}\right]-(2 \gamma+8 \Gamma) \rho_{41} \text {, } \\
& \dot{\rho}_{42}=-i\left[\left(H_{44}-H_{22}\right) \rho_{42}+H_{42}\left(\rho_{22}-\rho_{44}\right)+H_{41} \rho_{12}+H_{43} \rho_{32}-H_{12} \rho_{41}-H_{32} \rho_{43}\right]+2 \gamma \rho_{31}-(\gamma+4 \Gamma) \rho_{42} \text {, } \\
& \dot{\rho}_{43}=-i\left[\left(H_{44}-H_{33}\right) \rho_{43}+H_{43}\left(\rho_{33}-\rho_{44}\right)+H_{41} \rho_{13}+H_{42} \rho_{23}-H_{13} \rho_{41}-H_{23} \rho_{42}\right]+2 \gamma \rho_{21}-(\gamma+4 \Gamma) \rho_{43} \text {, } \\
& \dot{\rho}_{44}=-i\left[H_{14}\left(\rho_{14}-\rho_{41}\right)+H_{42} \rho_{24}+H_{43} \rho_{34}-H_{24} \rho_{42}-H_{34} \rho_{43}\right]+2 \gamma\left(\rho_{22}+\rho_{33}\right) \text {. }
\end{aligned}
$$


After the evolution of the system with the ramp time $t_{\text {ramp }}$ and the total measurement time $t_{\text {meas }}$, one can obtain the final polarization along the $y$ direction at $t_{f}=t_{\text {ramp }}+t_{\text {meas }}$ by tracing the final density matrix governed by Eqs. (A8) as

$$
\left\langle\sigma_{1}^{y}\right\rangle+\left\langle\sigma_{2}^{y}\right\rangle=\operatorname{Tr}\left[\rho\left(t=t_{f}\right) \cdot\left(\sigma_{y} \otimes \mathbf{I}_{2 \times 2}\right)\right]+\operatorname{Tr}\left[\rho\left(t=t_{f}\right) \cdot\left(\mathbf{I}_{2 \times 2} \otimes \sigma_{y}\right)\right]
$$

For simplicity in our simulations, we treat the evolution of the qubits in the whole measurement progress as free evolution under decoherence.

[1] K. von Klitzing, G. Dorda, and M. Pepper, Phys. Rev. Lett. 45, 494 (1980).

[2] D. C. Tsui, H. L. Stormer, and A. C. Gossard, Phys. Rev. Lett. 48, 1559 (1982); R. B. Laughlin, ibid. 50, 1395 (1983).

[3] M. V. Berry, Proc. R. Soc. London A 392, 45 (1984).

[4] D. J. Thouless, M. Kohmoto, M. P. Nightingale, and M. den Nijs, Phys. Rev. Lett. 49, 405 (1982).

[5] Q. Niu, D. J. Thouless, and Y. S. Wu, Phys. Rev. B 31, 3372 (1985).

[6] D. Xiao, M. C. Chang, and Q. Niu, Rev. Mod. Phys. 82, 1959 (2010).

[7] A. C. M. Carollo and J. K. Pachos, Phys. Rev. Lett. 95, 157203 (2005); S. L. Zhu, ibid. 96, 077206 (2006); X. Peng, S. Wu, J. Li, D. Suter, and J. Du, ibid. 105, 240405 (2010).

[8] E. Sjöqvist, Physics 1, 35 (2008).

[9] P. Zanardi and M. Rasetti, Phys. Lett. A 264, 94 (1999); L.-M. Duan, J. I. Cirac, and P. Zoller, Science 292, 1695 (2001).

[10] S. L. Zhu and Z. D. Wang, Phys. Rev. Lett. 89, 097902 (2002); 91, 187902 (2003); Z.-T. Liang, Y.-X. Du, W. Huang, Z.-Y. Xue, and H. Yan, Phys. Rev. A 89, 062312 (2014); Z.-Y. Xue, Quant. Info. Proc. 11, 1381 (2012).

[11] V. Gritsev and A. Polkovnikov, Proc. Natl. Acad. Sci. USA 109, 6457 (2012).

[12] J. E. Avron, M. Fraas, G. M. Graf, and O. Kenneth, New J. Phys. 13, 053042 (2011).

[13] I. M. Georgescu, S. Ashhab, and F. Nori, Rev. Mod. Phys. 86, 153 (2014); S. Ashhab, New J. Phys. 16, 113006 (2014).

[14] P. J. Leek, J. M. Fink, A. Blais, R. Bianchetti, M. Göppl, J. M. Gambetta, D. I. Schuster, L. Frunzio, R. J. Schoelkopf, and A. Wallraff, Science 318, 1889 (2007).

[15] A. A. Abdumalikov, Jr., J. M. Fink, K. Juliusson, M. Pechal, S. Berger, A. Wallraff, and S. Filipp, Nature (London) 496, 482 (2013).

[16] X. Tan, D. W. Zhang, Z. Zhang, Y. Yu, S. Han, and S. L. Zhu, Phys. Rev. Lett. 112, 027001 (2014); S. Gasparinetti, P. Solinas, and J. P. Pekola, ibid. 107, 207002 (2011).

[17] M. D. Schroer, M. H. Kolodrubetz, W. F. Kindel, M. Sandberg, J. Gao, M. R. Vissers, D. P. Pappas, A. Polkovnikov, and K. W. Lehnert, Phys. Rev. Lett. 113, 050402 (2014).
[18] P. Roushan, C. Neill, Y. Chen, M. Kolodrubetz, C. Quintana, N. Leung, M. Fang, R. Barends, B. Campbell, Z. Chen, B. Chiaro, A. Dunsworth, E. Jeffrey, J. Kelly, A. Megrant, J. Mutus, P. O’Malley, D. Sank, A. Vainsencher, J. Wenner, T. White, A. Polkovnikov, A. N. Cleland, and J. M. Martinis, Nature (London) 515, 241 (2014).

[19] R. A. Pinto, A. N. Korotkov, M. R. Geller, V. S. Shumeiko, and J. M. Martinis, Phys. Rev. B 82, 104522 (2010).

[20] G. S. Paraoanu, J. Low Temp. Phys. 175, 633 (2014).

[21] T. P. Orlando, J. E. Mooij, L. Tian, C. H. van der Wal, L. S. Levitov, S. Lloyd, and J. J. Mazo, Phys. Rev. B 60, 15398 (1999).

[22] A. Weiße and H. Fehske, Computational Many-Particle Physics, Lecture Notes in Physics Vol. 739 (Springer, Berlin, Heidelberg, 2008), p. 529.

[23] G. S. Agarwal, Quantum Optics (Cambridge University Press, Cambridge, UK, 2013).

[24] E. Lucero, R. Barends, Y. Chen, J. Kelly, M. Mariantoni, A. Megrant, P. O’Malley, D. Sank, A. Vainsencher, J. Wenner, T. White, Y. Yin, A. N. Cleland, and J. M. Martinis, Nat. Phys. 8, 719 (2012).

[25] J. D. Whittaker, F. C. S. da Silva, M. S. Allman, F. Lecocq, K. Cicak, A. J. Sirois, J. D. Teufel, J. Aumentado, and R. W. Simmonds, Phys. Rev. B 90, 024513 (2014).

[26] In fact in this coupled multiqubit system, there are more decoherence channels with shorter effective decoherence times than in the single-qubit system, which can be seen from the master equation, (A8), in the Appendix. When the open-system effects become significant, such as in this case, the Chern number from the Berry phase is no longer a well-defined topological index. To better characterize the topological property of an open (and thermal) system, one may consider the topological index via the Uhlmann phase. For example, see the following references: O. Viyuela, A. Rivas, and M. A. Martin-Delgado, Phys. Rev. Lett. 112, 130401 (2014); 113, 076408 (2014); Z. Huang and D. P. Arovas, ibid. 113, 076407 (2014).

[27] U. Patel, Y. Gao, D. Hover, G. J. Ribeill, S. Sendelbach, and R. McDermotta, Appl. Phys. Lett. 102, 012602 (2013).

[28] J. Bylander, S. Gustavsson, F. Yan, F. Yoshihara, K. Harrabi, G. Fitch, D. G. Cory, Y. Nakamura, J.-S. Tsai, and W. D. Oliver, Nat. Phys. 7, 565 (2011). 